\title{
EPILEPSY
}

Day 2, Friday 8 February

Speakers, Short Biographies and Abstracts

\section{CELLULAR MECHANISMS OF EPILEPSY}

doi:10.1136/jnnp-2013-306103.12

John Jefferys

John Jefferys FMedSc, Professor of Neuroscience, Neurotrauma and Neurodegeneration School of Clinical and Experimental Medicine, College of Medical and Dental Sciences, University of Birmingham, Edgbaston, Birmingham B15 2TT Email j.g.r. jefferys@bham.ac.uk

Epilepsy is a chronic disease. While anyone can experience the excessive neuronal activity of a seizure if exposed to convulsant conditions, persons with epilepsy have seizures with no obvious immediate cause. Essentially the functional organisation of the epileptogenic neural networks renders the brain of persons with epilepsy prone to have spontaneous, unpredictable, usually time-limited seizures. I will outline how focal seizures arise from networks of interconnected neurons, essentially as a "chain reaction", and how the properties of neurons, synapses and networks are changed in chronic epileptic foci. During the prolonged "interictal" periods between seizures, these cellular and network changes provide potential explanations for behavioural and other comorbidities of epilepsy. Finally I will discuss recent work on high frequency oscillations associated with epileptic foci and their potential application in defining epileptogenic zones for surgical resection.

Funded by MRC and Epilepsy Research UK. 